\title{
Study on Pagefile.sys in Windows System
}

\author{
Nisarg Trivedi \\ Institute of Forensic Science, Gujarat Forensic Sciences University, Gujarat, India
}

\begin{abstract}
Pagefile.sys is a file that is used by Microsoft Windows to store frames of memory that do not currently fit into physical memory. It means Windows uses a page file to store data that can't be held by your computer's random-access memory when it fills up. Analysis of the Pagefile.sys gives the information of which events were done on PC. Analysis of Pagefile.sys can give the sensitive information such as User Ids, Passwords, Hidden Processes, Download info, Search Activity of Browser etc. This Paper represents various approaches and tools used to capture and analyze data from Pagefile.sys.
\end{abstract}

Keywords: Physical Memory, Artifacts in Pagefile.sys, Sensitive information.

\section{Introduction}

Windows uses part of your hard drive space as "virtual memory" [7]. It loads what it needs to load into the much faster RAM (random access memory) memory, but creates a swap or page file on the hard drive that it uses to swap data in and out of RAM ${ }^{[7]}$. Pagefile.sys is located on the root of C: drive (or in where the Operating System is installed) and is named as pagefile.sys, but it is a hidden system file so you won't see it unless you have changed your file viewing settings to show hidden and system files ${ }^{[7]}$. Pagefile.sys is a windows system files, acts as swap file and was designed to improve performance ${ }^{[8]}$.

Virtual memory allows Windows to open more windows and run more programs simultaneously while only keeping the one being actively used in RAM ${ }^{[7]}$. The "problem" lies in the fact that information remains in the page file. As you use different programs and perform different functions on your computer the page file may end up containing all sorts of potentially sensitive or confidential information ${ }^{[7]}$. Event Log Records, like other data, may be found within the Pagefile or within unallocated space ${ }^{[1]}$.

\section{Myth about Pagefile.sys}

Disabling the Page File Improves Performance ${ }^{[3]}$

Some people will tell you that you should disable the page file to speed up your computer ${ }^{[3]}$. The thinking goes like this: the page file is slower than RAM, and if you have enough RAM, Windows will use the page file when it should be using RAM, slowing down your computer ${ }^{[3]}$.

This isn't really true. People have tested this theory and found that, while Windows can run without a page file if you have a large amount of RAM, there's no performance benefit to disabling the page file ${ }^{[3]}$.

However, disabling the page file can result in some bad things ${ }^{[3]}$. If programs start to use up all your available memory, they'll start crashing instead of being swapped out of the RAM into your page file ${ }^{[3]}$. This can also cause problems when running software that requires a large amount of memory, such as virtual machines ${ }^{[3]}$. Some programs may even refuse to run ${ }^{[3]}$.

In summary, there's no good reason to disable the page file - you'll get some hard drive space back, but the potential system instability won't be worth it ${ }^{[3]}$. Note that Cleaning the pagefile.sys at system shutdown increases the performance of the system ${ }^{[6]}$.

\section{Management of Pagefile.sys}

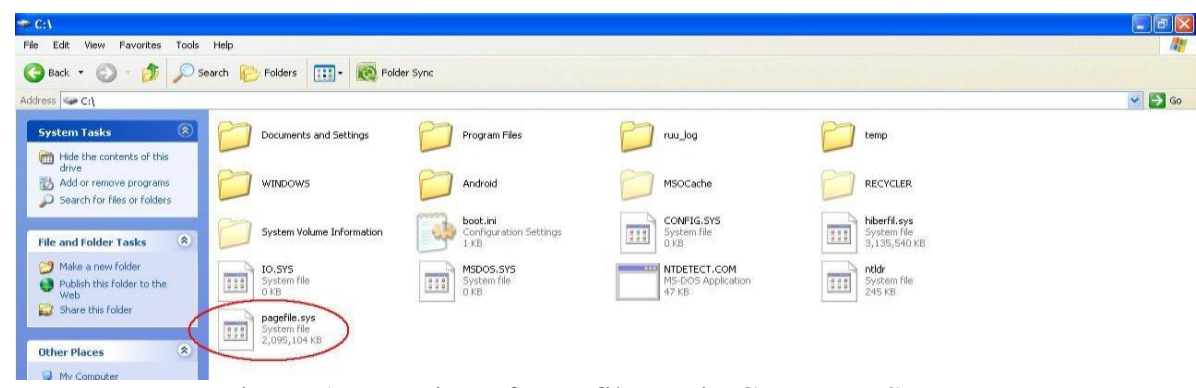

Figure 1: Location of Pagefile.sys in Computer System 
The page file isn't use consistently, so some data may linger there for quite some time ${ }^{[2]}$. Above Figure shows the location of Pagefile in User's Computer System. By default it is generated and Located in same in which the Operating System is installed (You can also change its location). The above Pagefile size is approximately $2.00 \mathrm{GiB}$. The RAM of the above system is $3.00 \mathrm{GiB}$.

In Windows XP and 2000, you can clean the file pagefile.sys to improve its overall performance. This can be done by creating or modifying the REG_DWORD value from 1 to 0 in the registry key ClearPageFileAtShutdown ${ }^{[6]}$. This is located here.

Right Click on MyComputer $\rightarrow$ Advance Option $\rightarrow$ Settings button of Performance $\rightarrow$ Select Advance $\rightarrow$ Virtual Memory $\rightarrow$ Change

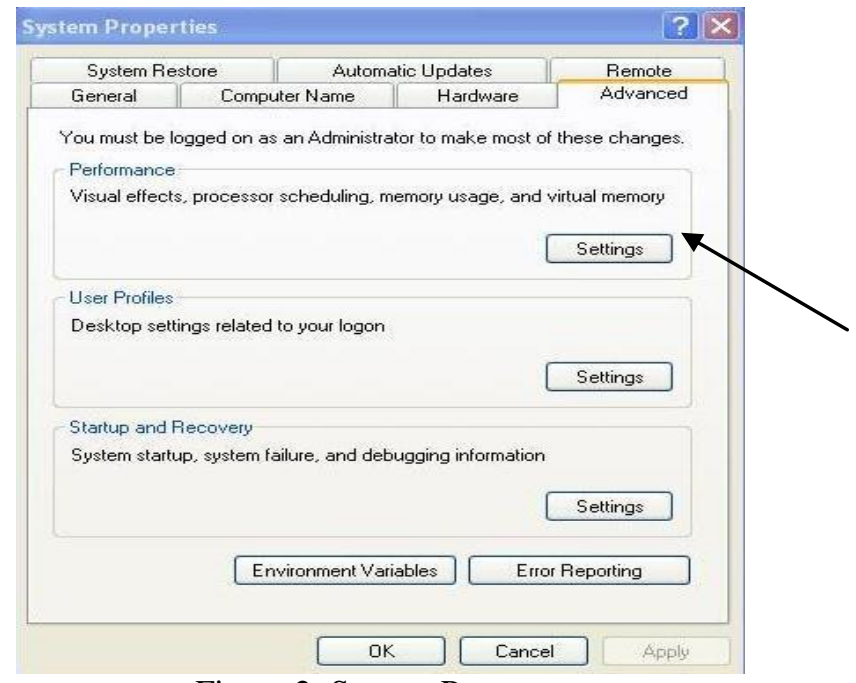

Figure 2: System Property

Click on the Setting Button.

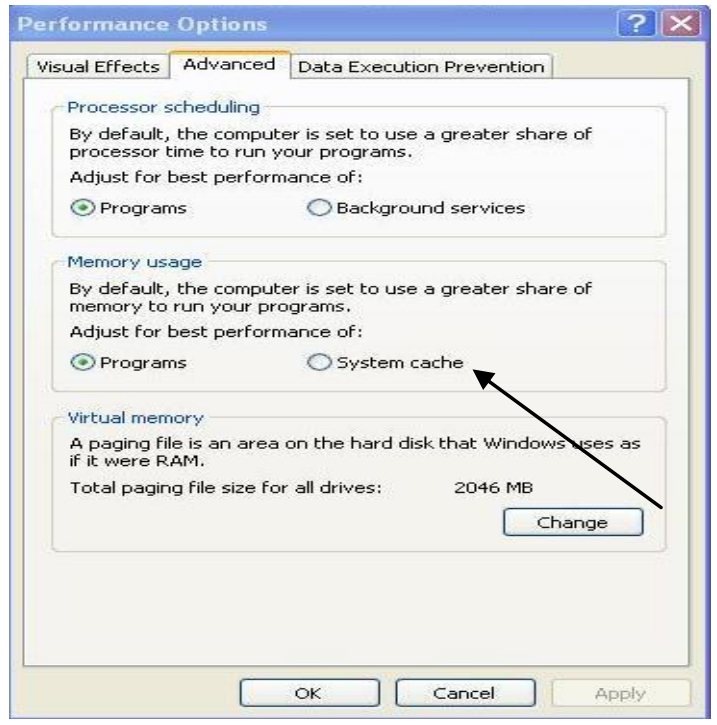

Figure 3: Performance Option

It must be noted that default size of virtual memory is $2046 \mathrm{MB}$, but with the help of change button, it can be varied as per requirement. 


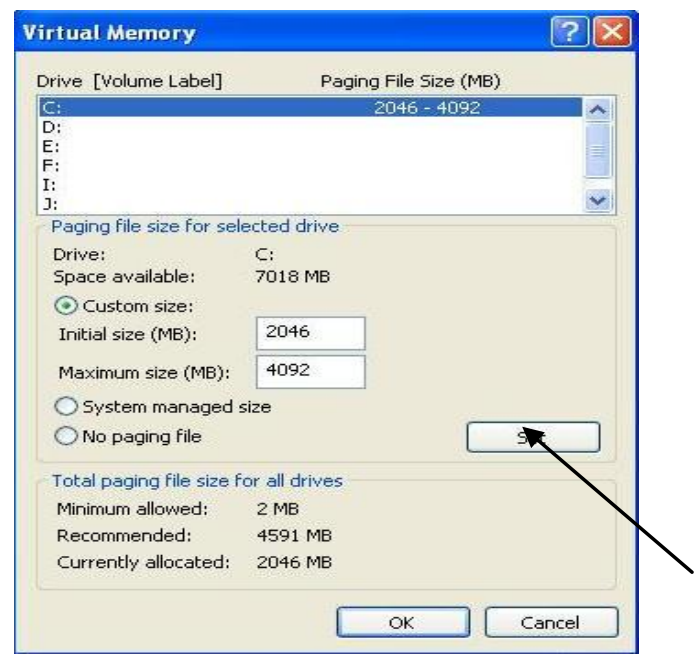

Figure 4: Virtual Memory

In above figure, it can be seen that Operating system is installed in $\mathrm{C}$ drive and hence default location of the pagefile.sys is also located in same.

Total paging file sizes for all drives are also mentioned which illustrate minimum allowed size, currently allocated as well as recommended size.

\section{Pagefile.sys Analysis}

Below screenshot is taken by using LinuxReader software ${ }^{[4]}$. We can read pagefile.sys by using this software. Information which can be gathered is mentioned below. Pagefile could be read in LinuxReader software in Text/Binary/Hex/Unicode etc.

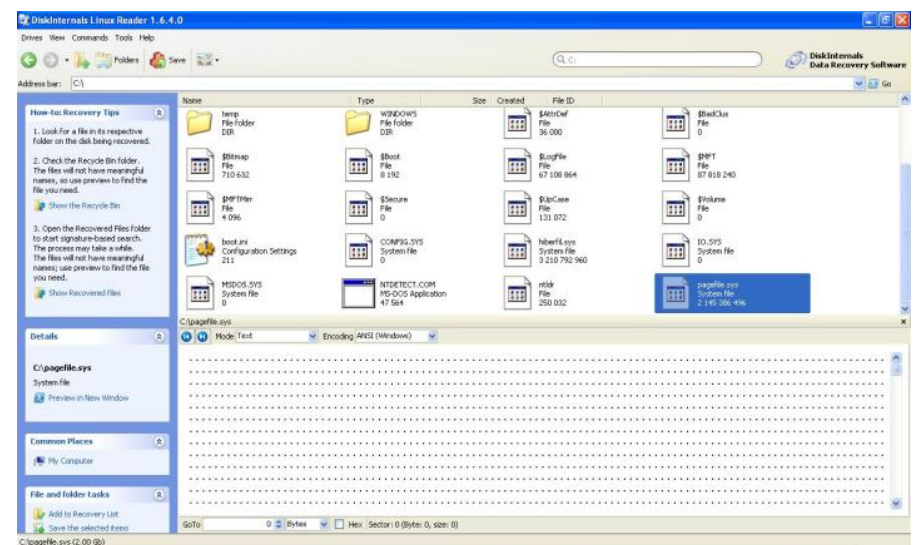

Figure 5: Open Pagefile.sys in Linux Reader Software

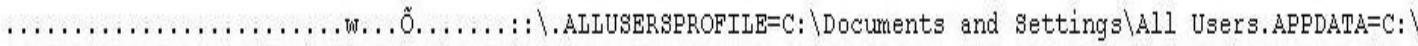
Documents and settings $\backslash$ Trivedi's $\backslash$ Application Data.CIIENTNAME=Console.CommonProgramFiles=C: $\backslash$ Program Files $\backslash$ Common Files.COMPUTERNAME=NISARG. ComSpec=C: \WINDOWS $\backslash$ system32 \amd. exe. FP_NO_HOST_CHECK=NO. HOMEDRIVE=C: . HOMEPATH= Documents and settings $\backslash$ Trivedi's. LOGONSERVER= \\ISARG. NUMBER_OF_PROCESSORSS=2.OS=Windows_NT. Path=C: \WINDOWS $\backslash$

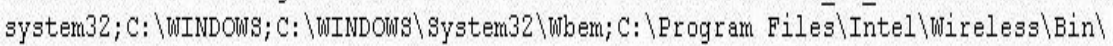

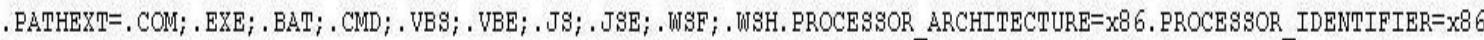
Family 6 Model 15 stepping 6, GenuineIntel.PROCESSOR_LEVEL=6. PROCESSOR_REVISION=0 f06.ProgramFiles=C: $\backslash$ Program Files. SESSIONNAME=Console. SystemDrive=C: . SystemRoot=C: \WINDOWS. TEMP=C: \DOCIME $\sim \backslash$ TRIVED $\sim 1 \backslash$ IOCALS $\sim 1 \backslash \mathrm{Temp}$. TMP=C: \ DOCIME $\sim \backslash$ TRIVED $\sim 1 \backslash$ LOCALS $\sim 1 \backslash$ Temp. USERDOMAIN=NISARG. USERNAME=Trivedi' $s$. USEREROFILE=C: \Documents and settings $\backslash$ Trivedi's, windir $=\mathrm{C}: \backslash$

Figure 6: Information about Computer in LinuxReader Software

This screenshot gives information about our computer such as, Name of Computer, Number of Processor, Processor Architecture, Processor Level. 


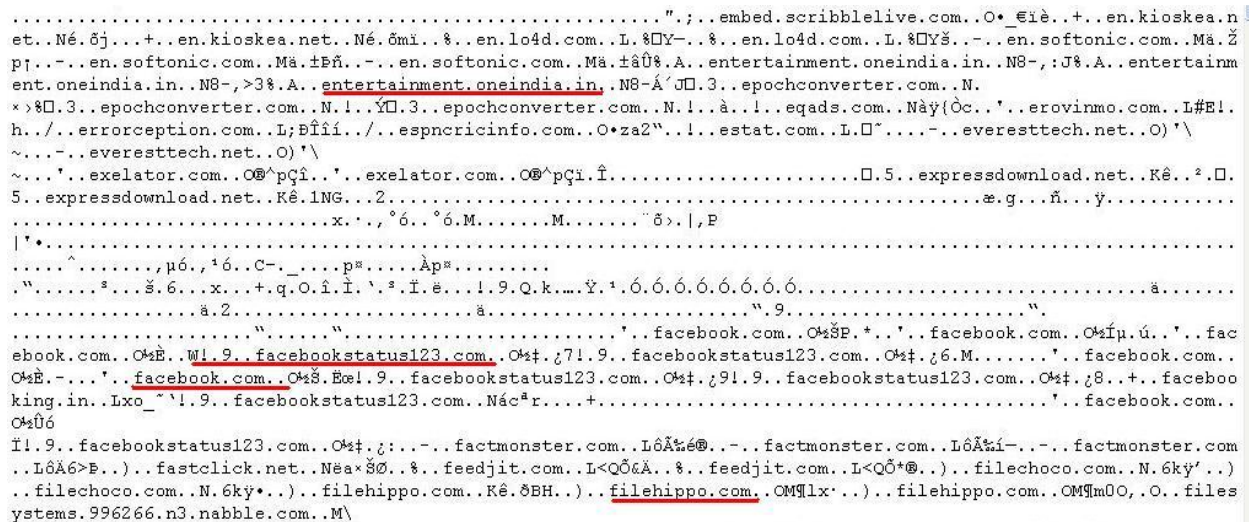

Figure 7: Information about Web History in LinuxReader Software

This screenshot describe web history visited through the web browser by user. It can be seen in figure that underlined areas describes links of the websites that have been visited by user through web browser. Other text in figure encrypted form, not readable by examiner. There is also some Useless stuff can be seen in the figure.

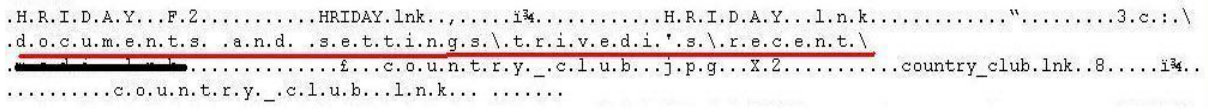

Figure 8: Information about Recent Data in LinuxReader Software

Recent document opened by user is underlined. From Pagefile.sys we can find the recent activity done by the User. Recently visited Folders and Files can be found by investigating Pagefile.sys. It will give all the information of User's visited file or folder on the System.

$\mathrm{HxD}$ is a hex editor, disk editor, and memory editor for Windows. It can open files larger than $4 \mathrm{GiB}$ and open and edit the raw contents of disk drives, as well as display and edit the memory used by running processes. It can calculate various checksums, compare files, or shred files.

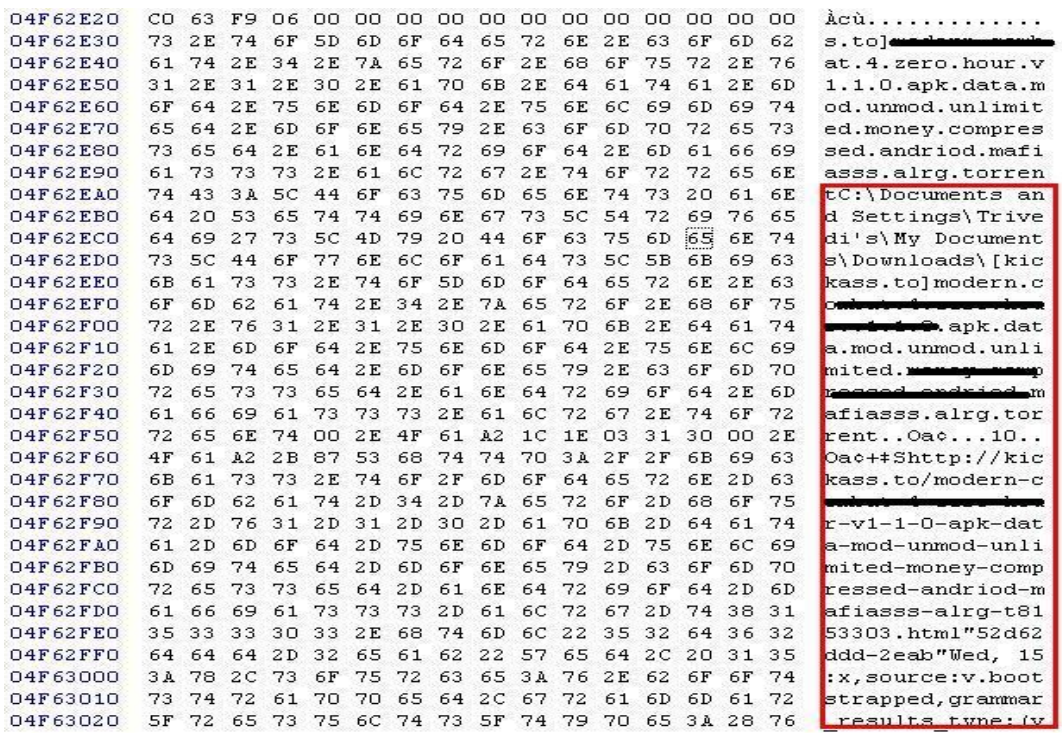

Figure 9: Downloaded Data Information using HxD Software

This screenshot is taken by using HxD Software ${ }^{[5]}$. Downloaded items and its file location with its full path can be seen in the screenshot. Here the downloaded apk file stored in Downloads Folder located in My Documents of C: drive. All the downloaded data link can be retrieved in Pagefile.sys. 


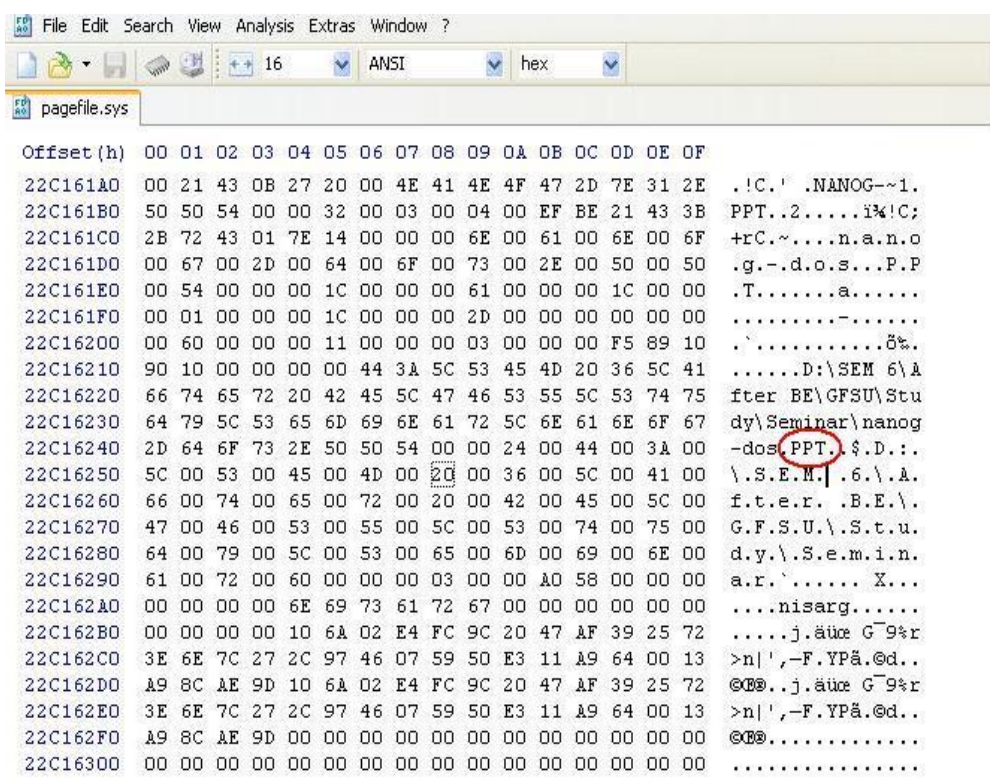

Figure 10: Extension Information using HxD Software

In above figure, it can be seen that we locate a specific file through its extension by entering in a search dialogue box. For example, in above figure ".ppt" has been entered as search criteria and all the files of this extension are achieved. U can also enter "crime" "terrorist" "gun" "hack" etc. (words may be varies through case by case) to check whether there is any file or information related to crime is exist there or not.

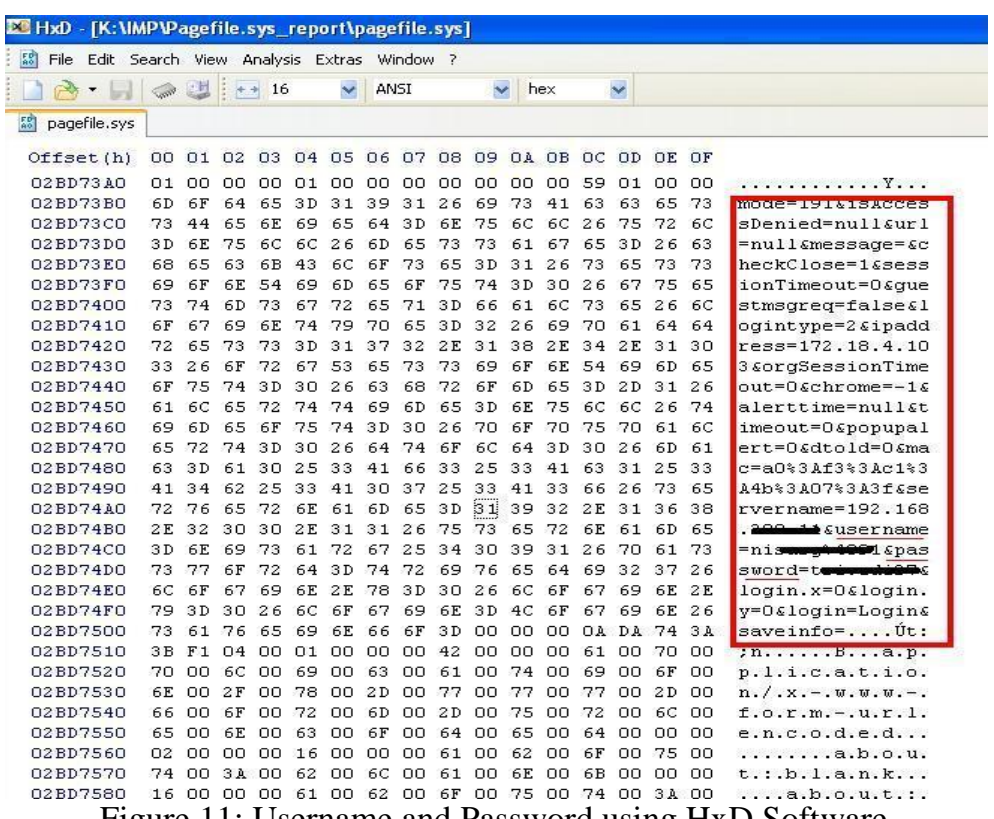

Figure 11: Username and Password using HxD Software

Using this tool we can find lots of sensitive information. I have found the login in to some account which shows the username and password. Like this e-mail addresses could also found. 


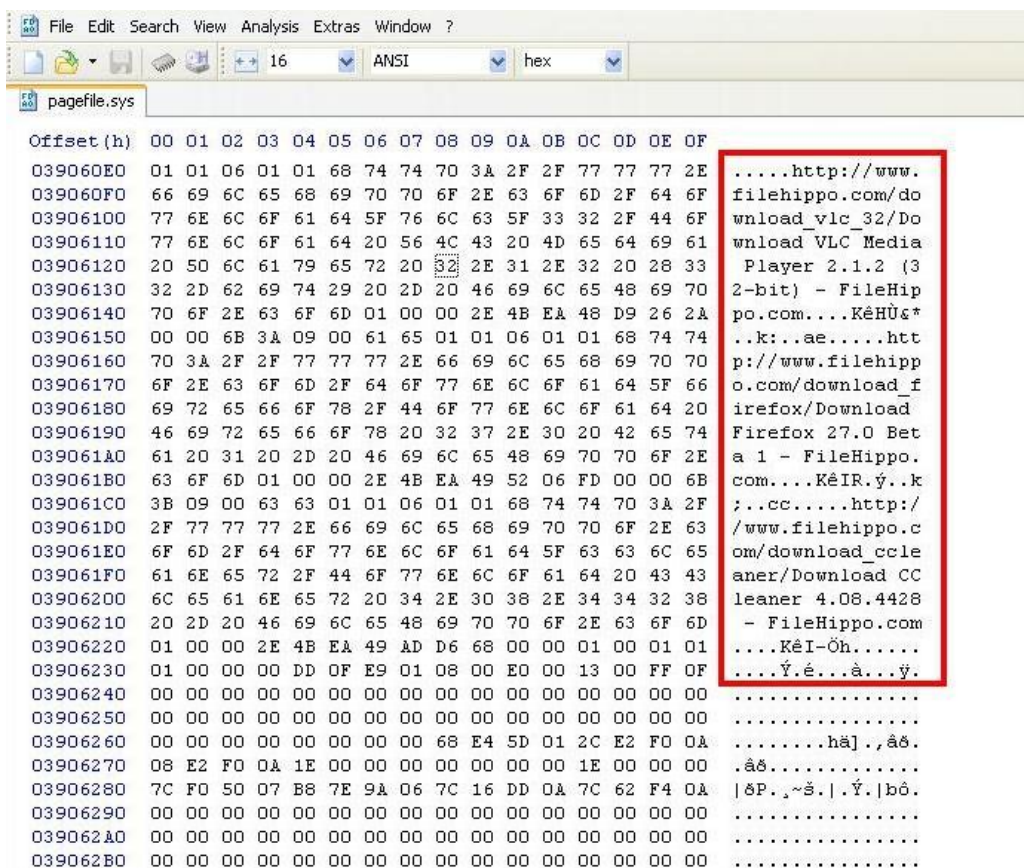

Figure 12: Downloaded Software Information using HxD Software

Above figure shows the download of software from the web link. Software with its version which is downloaded in the system can be found. Like this we can find.

These all are the information which we can get through different tools. The tools which are used for the analysing the Pagefile.sys have their different approach. The above tools are the graphical tools, there are some other tools which also do the Pagefile.sys analysis.

\section{Conclusion}

There are so many tools and techniques are available for Pagefile.sys analysis. They all have different methods and different approaches. It is very good to find out the sensitive information from the Pagefile.sys. This is helpful for solving the many crimes related to computer. The data which is stored in the Pagefile.sys are changes repeatedly. Pagefile.sys is also useful for investigation purpose for obtaining information. The tools which are used for analysis have to be developing more powerful with coming years.

\section{References}

[1] Harly Carvey, "Windows Forensic Analysis Toolkit"

[2] John Sammons, "The Basics of Digital Forensic"

[3] http://www.howtogeek.com/126430/htg-explains-what-is-the-windows-page-file-and-should-you-disable-it/

[4] Linux Reader Software http://www.freewarefiles.com/DiskInternals-Linux-Reader program 21787.html

[5] HxD Software http://mh-nexus.de/en/hxd/ .

[6] http://en.kioskea.net/faq/719-pagefile-sys-file

[7] http://netsecurity.about.com/od/windowsxp/qt/aa071004.htm

[8] http://www.neuber.com/taskmanager/process/pagefile.sys.html 